\title{
TITLE:
}

\section{Inverse Schrodinger equation and the exact wave function}

\author{
$\operatorname{AUTHOR}(S)$ :
}

Nakatsuji, $\mathrm{H}$

\section{CITATION:}

Nakatsuji, H. Inverse Schrodinger equation and the exact wave function. PHYSICAL REVIEW A 2002, 65(5): 052122.

ISSUE DATE:

2002-05

URL:

http://hdl.handle.net/2433/39822

RIGHT:

Copyright 2002 American Physical Society 
PHYSICAL REVIEW A, VOLUME 65, 052122

\title{
Inverse Schrödinger equation and the exact wave function
}

\author{
Hiroshi Nakatsuji \\ Department of Synthetic Chemistry and Biological Chemistry, Graduate School of Engineering, Kyoto University, \\ Sakyo-ku, Kyoto 606-8501, Japan
}

(Received 17 December 2001; published 10 May 2002)

\begin{abstract}
Using the inverse of the Hamiltonian, we introduce the inverse Schrödinger equation (ISE) that is equivalent to the ordinary Schrödinger equation (SE). The ISE has the variational principle and the $H$-square group of equations as the SE has. When we use a positive Hamiltonian, shifting the energy origin, the inverse energy becomes monotonic and we further have the inverse Ritz variational principle and cross- $H$-square equations. The concepts of the SE and the ISE are combined to generalize the theory for calculating the exact wave function that is a common eigenfunction of the SE and ISE. The Krylov sequence is extended to include the inverse Hamiltonian, and the complete Krylov sequence is introduced. The iterative configuration interaction (ICI) theory is generalized to cover both the SE and ISE concepts and four different computational methods of calculating the exact wave function are presented in both analytical and matrix representations. The exact wave-function theory based on the inverse Hamiltonian can be applied to systems that have singularities in the Hamiltonian. The generalized ICI theory is applied to the hydrogen atom, giving the exact solution without any singularity problem.
\end{abstract}

DOI: 10.1103/PhysRevA.65.052122

PACS number(s): 03.65.Ca, 03.65.Ge, 31.10.+z, 31.15.-p

\section{INTRODUCTION}

The basic principles of chemistry are described by the Schrödinger equation (SE) and the corresponding relativistic equation [1]. Solving these equations as accurately as possible is, therefore, the central theme of theoretical chemistry. In this series of papers [2-6], our aim is to formulate a systematic method of solving the SE, and for this purpose, we have studied the structure of the exact wave function. Since the exact wave function is an eigenfunction of the Hamiltonian whose structure is very simple - a sum of only one- and two-particle operators, such as

$$
H=\sum_{i}-\frac{1}{2} \Delta_{i}-\sum_{i} \sum_{A} Z_{A} / r_{A i}+\sum_{i>j} 1 / r_{i j}
$$

for atomic and molecular systems, we expect that the exact wave function must also have a simple structure, reflecting the simplicity of the structure of the Hamiltonian. When the structure of the exact wave function is clarified to be simple, we would be able to find an easier way of solving the exact wave function than before. We have proposed the iterative configuration-interaction (ICI) $[2,3]$ and extended coupled cluster (ECC) [4] methods to calculate the exact wave function. The methods of calculating excited states based on these theories are formulated [3,5], and the applications of these theories to some simple system $[4,5]$ and to the atomic and molecular system [6] have been given. Van Voorhis and Head-Gordon [7] have reported that the coupled cluster general singles and doubles (CCGSD) method, which is a special case of the ECC method, has reproduced the full-CI result for $\mathrm{Ne}$ and $\mathrm{N}_{2}$. We have shown more recently that for many atoms and molecules, the ICI method reproduces the full-CI results with only a few (one to three) variables [6], in contrast to a very large number of variables in the conventional full-CI algorithm [8].
Another approach for solving the SE is due to the densitymatrix theory (DMT) [9]. Since all the elementary physical properties can be calculated from the second-order density matrix $\Gamma^{(2)}$, we may utilize it as a basic variable of quantum mechanics instead of the wave function $\psi$. Calculating $\Gamma^{(2)}$ through the variational principle with some $\mathrm{N}$-representability conditions $[10,11]$ has long been a subject of discussion $[12,13]$ since a birth of the DMT. Recently, a general computational method of this density-matrix variational theory (DMVT) has been developed [14] by using the positive-semidefinite programming algorithm, and a high potentiality of the DMVT has been demonstrated [15].

Another method in the DMT is to first derive the equation that is equivalent to the SE but contains only density matrices as variables. The density equation (DE) [16] is such an equation and, therefore, we call this approach the density equation theory (DET). Later, this equation was alternatively called the contracted Schrödinger equation [17]. To solve this equation, we need relations between $\Gamma^{(3)}, \Gamma^{(4)}$, and $\Gamma^{(1)}$, $\Gamma^{(2)}$. Valdemoro and co-workers [18] proposed approximate relations, we proposed the improved relations using the Green's function method [19], and Mazziotti [20] gave the relations using the cumulant expansion method. We have calculated the ground state of many atoms and molecules to good accuracy by the DET [19]. Recently, many studies have been presented for the further development of the science lying here $[8,21]$. In particular, the cumulant expansion method [22] has been shown to be useful to systematize the relations among the density matrices in different orders and to develop further this theory [23]. Nooijen [24] considered solving the CCGSD using the DE, which circumvents the $N$-representability problem.

Certainly, the DMVT and the DET are very promising. It is, however, true that an obstacle in these approaches still lies in our limited knowledge on the $\mathrm{N}$-representability condition [10]. 
In this paper, we take the wave-function approach. We first introduce the inverse Schrödinger equation (ISE) that is completely equivalent to the SE. The ISE has the same determinative power as the ordinary SE. Further, similar to the ordinary SE, the ISE has the variational principle and the $\mathrm{H}$-square [25] group of equations, which are equivalent to the ISE. The cross- $H$-square equation couples the two worlds of the SE and the ISE. Based on these observations, we continuously develop the method of calculating the exact wave functions [2-6] for general systems such as atomic and molecular systems. The ICI and the ECC theories are equally defined for the ISE and are generalized to cover both the SE and ISE concepts.

The ICI and the ECC theories utilize the products of the Hamiltonian or the divided Hamiltonian applied to an appropriate reference function to construct the exact wave function. However, when the Hamiltonian includes singularities such as those by the nuclear attraction operator and the electron repulsion operator in the atomic and molecular Hamiltonian given by Eq. (1.1), their higher-order products applied to the reference function do not satisfy the quantummechanical condition that the physical wave function must satisfy, i.e., integrable finiteness. The singularity of the nuclear attraction operator is too large to be solved by the finite size of the nucleus [26,27]. This problem is similar to those encountered in the analytical formulations of the $t$-expansion method [28] and the connected-moments method [29]. This difficulty was also pointed out [30] to occur in the application of the surplus function method [31], which is similar to the simplest case of the ICI theory [3].

However, in such a case, an introduction of the inverse Hamiltonian of the ISE resolves the problem. This is shown by taking the Krylov sequence [32] as an example: by extending the Krylov sequence into the inverse side of the Hamiltonian, we have a sequence that naturally satisfies the quantum-mechanical condition. The ICI theory is generalized based on both the SE and ISE concepts and by the $H$-square and the cross- $H$-square group of equations. Four different formulas of the generalized ICI (GICI) theory are presented, both in the analytical form and in matrix representation. The generalized ICI theory is useful even for systems that have singularities in the Hamiltonian. Numerical examples are given by applying the present theory to the hydrogen atom, which has a Coulombic singularity at the position of the nucleus. The concluding remarks are given in Sec. VII.

\section{INVERSE SCHRÖDINGER EQUATION}

Suppose that we have an eigenvalue problem

$$
\mathbf{A C}=\mathbf{C E},
$$

where $\mathbf{A}$ is a regular Hermitian matrix and $\mathbf{E}$ and $\mathbf{C}$ are its eigenvalue and eigenvector. When we introduce the inverse of $\mathbf{A}, \mathbf{A}^{-1}$ as

$$
\mathbf{A}^{-1} \mathbf{A}=\mathbf{A} \mathbf{A}^{-1}=\mathbf{1},
$$

then it is easy to show that the inverse of $\mathbf{A}$ satisfies

$$
\mathbf{A}^{-1} \mathbf{C}=\mathbf{C E}^{-1} .
$$

Namely, the eigenvalue of the inverse matrix is the inverse of the eigenvalue of the original matrix, and the eigenvector is common to $\mathbf{A}$ and $\mathbf{A}^{-1}$.

We apply this relation to the Schrödinger equation (SE),

$$
H \psi=E \psi
$$

Based on Eq. (2.2), we define the inverse of the Hamiltonian $H^{-1}$ as

$$
H^{-1} H=H H^{-1}=1,
$$

then it is easy to show that $H^{-1}$ satisfies

$$
H^{-1} \psi=E^{-1} \psi,
$$

which is called the inverse Schrödinger equation (ISE). Conversely, we can obtain SE from ISE by using Eq. (2.5). Thus, on the assumption that the inverse Hamiltonian exists, the $S E$ and the ISE are equivalent.

Note that $H$ and $H^{-1}$ commute, as easily seen from Eq. (2.5). As the Hamiltonian is an operator, including differential operators, etc., it is not necessarily straightforward to write down the analytical form of the inverse Hamiltonian. However, quantum mechanically, we can always define the matrix form of the Hamiltonian $\mathbf{H}$, and then we can always define its inverse $\mathbf{H}^{-1} . H^{-1}$ may be defined as the analytical correspondence of the inverse matrix $\mathbf{H}^{-1}$. In the matrix formulation of quantum mechanics [33] $\mathbf{H}$ and $\mathbf{H}^{-1}$ are entirely equivalent.

Hereafter, we call $H$ as regular Hamiltonian or simply ordinary Hamiltonian to distinguish it from the inverse Hamiltonian.

Here, a remark is necessary on the inverse energy. The energy $E$ in Eq. (2.4) may be defined by

$$
\langle\psi|H-E| \psi\rangle=0,
$$

namely, by

$$
E=\langle\psi|H| \psi\rangle /\langle\psi \mid \psi\rangle .
$$

The inverse of the energy $E^{-1}$ is, therefore,

$$
E^{-1} \equiv 1 / E=\langle\psi \mid \psi\rangle /\langle\psi|H| \psi\rangle .
$$

On the other hand, from Eq. (2.6), we obtain $\langle\psi| H^{-1}$ $-E^{-1}|\psi\rangle=0$, which gives

$$
E^{-1}=\left\langle\psi\left|H^{-1}\right| \psi\right\rangle /\langle\psi \mid \psi\rangle .
$$

For the exact wave function $\psi$, two expressions, Eqs. (2.9) and (2.10), are equivalent, but for approximate ones, they are different. For this reason, we introduce the inverse energy ${ }^{i} E$ associated with a wave function $\phi$ as

$$
{ }^{i} E[\phi] \equiv\left\langle\phi\left|H^{-1}\right| \phi\right\rangle /\langle\phi \mid \phi\rangle .
$$


If $\phi$ is not exact, the inverse energy ${ }^{i} E[\phi]$ is different from the inverse of the energy $E[\phi]$, which is defined as $\langle\phi| H$ $-E[\phi]|\phi\rangle=0$ similarly to Eq. (2.7). For the exact wave function $\psi$, we have

$$
{ }^{i} E[\psi]=E[\psi]^{-1} .
$$

\section{EQUATIONS EQUIVALENT TO SE AND ISE}

The equation that is equivalent to the SE is very important, since it has the same determinative power as the SE. The variational equation and the $H$-square group of equations [25] are two important equations that are equivalent to the SE. The variational equation is written as

$$
\langle\psi|H-E| \delta \psi\rangle=0
$$

and the Ritz variational principle for the ground state is given by

$$
E[\psi] \geqslant E_{g},
$$

where $E[\psi]$ is defined by

$$
\langle\psi|H-E[\psi]| \psi\rangle=0 .
$$

The simplest $H$-square equations [2] are given by

$$
\begin{gathered}
\left\langle\psi\left|(H-E)^{2}\right| \psi\right\rangle=0, \\
\langle\psi|(H-E) H| \psi\rangle=0,
\end{gathered}
$$

and

$$
\left\langle\psi\left|H^{2}-E^{2}\right| \psi\right\rangle=0,
$$

where $E$ of Eqs. (3.5) and (3.6) is defined by Eq. (3.3). For Eq. (3.4), such a definition of $E$ is unnecessary. More general $\mathrm{H}$-square equations were introduced in Paper II (Ref. [3]) as follows. We define a division of the Hamiltonian into $N_{D}$ parts as

$$
H=\sum_{I}^{N_{D}} H_{I}
$$

then the $H$-square equation is given, in general, by

$$
\left\langle\psi\left|(H-E)\left(H_{I}-E_{I}\right)\right| \psi\right\rangle=0
$$

or

$$
\left\langle\psi\left|(H-E) H_{I}\right| \psi\right\rangle=0
$$

for all $I\left(I=1, \ldots, N_{D}\right)$. In Eq. (3.8), $E_{I}$ is defined by $\langle\psi| H_{I}$ $-E_{I}|\psi\rangle=0$.

The variational equation is a flexible equation and is used to calculate the best possible wave function within a given functional form of $\psi$. The $H$-square equation is, on the other hand, a rather strict equation that is valid only for the exact wave function. Therefore, if a variation of a given $\psi$, substituted into the variational equation, leads to the $H$-square equation, then, that $\psi$ is guaranteed to have the structure of the exact wave function. Namely, in this case, the variational best is the exact wave function. We used this criterion to investigate the structure of the exact wave function [2-5].

We formulate here the equations that are equivalent to the ISE and therefore to the SE. They are useful not only as they are, but also for calculating the exact wave function. We first prove the inverse variational principle.

Theorem. When we define the inverse energy ${ }^{i} E[\psi]$ by

$$
\left\langle\psi\left|H^{-1}-{ }^{i} E[\psi]\right| \psi\right\rangle=0,
$$

then the wave function $\psi$ that satisfies

$$
\delta^{i} E[\psi]=0
$$

is exact in the necessary and sufficient sense.

Proof. Taking the derivative of Eq. (3.10), we get

$$
\left\langle\delta \psi\left|H^{-1}-{ }^{i} E\right| \psi\right\rangle+\left\langle\psi\left|H^{-1}-{ }^{i} E\right| \delta \psi\right\rangle-\langle\psi \mid \psi\rangle \delta^{i} E=0,
$$

where ${ }^{i} E$ represents ${ }^{i} E[\psi]$. From $\langle\psi \mid \psi\rangle \neq 0$ and Eq. (3.11), we obtain

$$
\left\langle\delta \psi\left|H^{-1}-{ }^{i} E\right| \psi\right\rangle+\left\langle\psi\left|H^{-1}-{ }^{i} E\right| \delta \psi\right\rangle=0,
$$

which leads to [34] the inverse variational equation

$$
\left\langle\psi\left|H^{-1}-{ }^{i} E\right| \delta \psi\right\rangle=0 .
$$

For arbitrary $\delta \psi$, Eq. (3.14) gives

$$
\left(H^{-1}-{ }^{i} E\right)|\psi\rangle=0,
$$

where ${ }^{i} E=E^{-1}$ for the uniqueness of the ISE. So, the sufficiency is proved. Conversely, when we have the ISE, ${ }^{i} E$ $=E^{-1}$ from Eq. (2.10) and we have Eq. (3.11) from Eq. (3.12). Q.E.D.

The Ritz variational principle can be inverted when the Hamiltonian has only positive eigenvalues. Though the ordinary Hamiltonian may have positive and negative eigenvalues, it is easily made a positive operator, $H_{p}$, by shifting the origin of the Hamiltonian, i.e., by adding some positive constant to the original Hamiltonian as

$$
H_{p}=H+\varepsilon_{c},
$$

where $\varepsilon_{c}$ is some positive constant with the dimension of energy. In the SE of $H_{p}$,

$$
H_{p} \psi=E_{p} \psi,
$$

and in the ISE,

$$
H_{p}^{-1} \psi=E_{p}^{-1} \psi
$$

the energy $E_{p}$ is

$$
E_{p}=E+\varepsilon_{c},
$$

which is positive by definition. Then, it is easy to show that for the ground state, the inverse positive energy ${ }^{i} E_{p}[\phi]$ associated with an approximate $\phi$, 


$$
\left\langle\phi\left|H_{p}^{-1}-{ }^{i} E_{p}[\phi]\right| \phi\right\rangle=0,
$$

gives a lower bound of the inverse of the positive energy $E_{p g}{ }^{-1}$ of the true ground state

$$
{ }^{i} E_{p}[\phi] \leqslant E_{p g}^{-1} .
$$

We call this relation the inverse Ritz variational principle for a positive Hamiltonian. Note that the subscript $g$ means the ground state.

Proof. We assume that we have the exact wave functions for the positive Hamiltonian as

$$
\begin{gathered}
H_{p} \psi_{K}=E_{p K} \Psi_{K}, \\
H_{p}^{-1} \Psi_{K}=E_{p K}^{-1} \Psi_{K} .
\end{gathered}
$$

Since $\left\{\Psi_{K}\right\}$ is a complete set, we expand $\phi$ of Eq. (3.20) by this set as $\phi=\Sigma_{K} C_{K} \Psi_{K}$ and obtain

$$
{ }^{i} E_{p}[\phi]-E_{p g}^{-1}=(\langle\phi \mid \phi\rangle)^{-1}\left\{\sum_{K}\left|C_{K}\right|^{2}\left(E_{p K}^{-1}-E_{p g}^{-1}\right)\right\} \leqslant 0,
$$

where the last inequality arises because $E_{p K}{ }^{-1}$ is always smaller than or equal to $E_{p g}{ }^{-1}$. The equality occurs when all $C_{K}=0$ except for $K=0=g$, namely, when $\phi=C_{0} \Psi_{0}$. Q.E.D.

Thus, in iterative variational calculations of the inverse positive energy, the inverse energy approaches from below the exact inverse energy. We further note that if the Hamiltonian is not positive, we do not have the inverse Ritz variational principle given above and, therefore, we cannot expect the above behavior in an iterative process.

The above argument has clarified the necessity of introducing the positive Hamiltonian when we introduce the inverse Hamiltonian. The reason is simple. Consider a hydrogen atom, then the eigenvalues of the regular Hamiltonian range from -0.5 to 0 for the bound states and 0 to $\infty$ for the unbound continuum states. Then, the eigenvalues of the corresponding inverse Hamiltonian range from -0.2 to $-\infty$ for the bound states and from $+\infty$ to 0 for the unbound continuum states. Thus, there exists a large discontinuous gap from $-\infty$ to $+\infty$ in the inverse energy spectrum. This is undesirable and can be easily avoided by shifting the origin of the energy of the regular Hamiltonian into the positive region. By shifting the regular Hamiltonian by unity to the positive side, i.e., $\varepsilon_{c}=1$, the eigenvalues of the bound states of the positive Hamiltonian range from 0.5 to 1.0 and those of the unbound states range from 1.0 to $+\infty$. Then, the eigenvalue spectrum of the inverse positive Hamiltonian ranges from 2.0 to 1.0 for the bound states and from 1.0 to 0.0 for the unbound states: they are monotonic and there is no infinite gap. Thus, when we shift the regular Hamiltonian into the positive region, we have monotonic descriptions of the energy eigenvalue spectra for both the regular and inverse Hamiltonians.

Next, we prove the inverse $H$-square theorem.

Theorem. We define the inverse $H$-square equation or $H^{-1}$-square equation by

$$
\left\langle\psi\left|\left(H^{-1}-{ }^{i} E\right)^{2}\right| \psi\right\rangle=0,
$$

then this equation holds if and only if $\psi$ satisfies the ISE.

Proof. When the ISE holds, $\psi$ is exact and Eq. (3.25) is valid when ${ }^{i} E=E^{-1}$. Conversely, when Eq. (3.25) holds, we define

$$
\Delta=\left(H^{-1}-{ }^{i} E\right) \psi,
$$

and then Eq. (3.25) implies $\langle\Delta \mid \Delta\rangle=0$, which is valid only when $\Delta=0$, namely,

$$
\left(H^{-1}-{ }^{i} E\right) \psi=0 .
$$

From the uniqueness of the ISE, ${ }^{i} E=E^{-1}$, namely, Eq. (3.27) is the ISE. Q.E.D.

With the definition of ${ }^{i} E={ }^{i} E[\psi]$ given by Eq. (3.10), it is easy to show that the following equations:

$$
\left\langle\psi\left|\left(H^{-1}-{ }^{i} E\right) H^{-1}\right| \psi\right\rangle=0
$$

and

$$
\left\langle\psi\left|\left(H^{-1}\right)^{2}-\left({ }^{i} E\right)^{2}\right| \psi\right\rangle=0
$$

are also equivalent to the ISE. Note that for the original $H^{-1}$-square theorem given by Eq. (3.25), ${ }^{i} E=E^{-1}$ is automatic and, therefore, Eq. (3.10) is unnecessary.

Similarly to Theorem II-1 of Paper II, we can prove the following $H^{-1}$-square theorem for the partitioned inverse Hamiltonian. Namely, when the inverse Hamiltonian is divided into $N_{D}$ (number of divisions) parts,

$$
H^{-1}=\sum_{I}^{N_{D}} d_{I}{ }^{i} H_{I},
$$

where $d_{I}$ is a constant, then the wave function $\psi$ that satisfies the following partitioned inverse $H$-square equation:

$$
\left\langle\psi\left|\left(H^{-1}-{ }^{i} E\right)\left({ }^{i} H_{I}-{ }^{i} E_{I}\right)\right| \psi\right\rangle=0
$$

or

$$
\left\langle\psi\left|\left(H^{-1}-{ }^{i} E\right)^{i} H_{I}\right| \psi\right\rangle=0
$$

for all $I\left(I=1, \ldots, N_{D}\right)$ with ${ }^{i} E$ defined by Eq. (3.10) and ${ }^{i} E_{I}$ defined by

$$
\left\langle\psi\left|{ }^{i} H_{I}-{ }^{i} E_{I}\right| \psi\right\rangle=0
$$

is exact in the necessary and sufficient sense. The partitioned inverse energy ${ }^{i} E_{I}$ satisfies

$$
\sum_{I} d_{I}{ }^{i} E_{I}={ }^{i} E
$$

Proof. When ISE holds, both Eqs. (3.31) and (3.32) hold, where ${ }^{i} E$ is given by Eq. (3.10). Conversely, when Eq. (3.31) or Eq. (3.32) holds, we multiply it by $d_{I}$, sum up for all $I$, and using Eqs. (3.30) and (3.34), we obtain Eq. (3.25) or Eq. (3.28), so that this $\psi$ is exact. Q.E.D. 
Note that in the above division of the inverse Hamiltonian given by Eq. (3.30), we have given the coefficients $d_{I}$ on each partitioned Hamiltonian ${ }^{i} H_{I}$. Throughout the proof, however, we need not to know the actual value of $d_{I}$. This means that all we must know for the division of the Hamiltonian is the individual functional forms of the operators ${ }^{i} H_{I}$, and not their coefficients. The same is true for the $\mathrm{H}$-square theorem of the divided Hamiltonian given in $\mathrm{Pa}-$ pers I and II. This relaxes the necessary knowledge on the regular and inverse Hamiltonians and would be useful when the detailed form of the Hamiltonian is difficult to know.

We now show an important $H$-square equation that combines the regular and inverse worlds of the Hamiltonian: we call it the cross-H-square theorem, which is valid only for the positive Hamiltonian. Namely, the following cross- $H$ square equation:

$$
\left\langle\psi\left|\left(H_{p}-E_{p}\right)\left(H_{p}^{-1}-E_{p}^{-1}\right)\right| \psi\right\rangle=0
$$

is equivalent to the SE and ISE in the necessary and sufficient sense.

Proof. When the SE and the ISE of the positive Hamiltonian are satisfied by $\psi$, Eq. (3.35) holds automatically. So, the necessity is evident. The sufficiency is as follows. We expand $\psi$ by the exact eigenfunctions, $\psi=\Sigma_{K} C_{K} \Psi_{K}$, and insert it into Eq. (3.35) and obtain

$$
\begin{aligned}
\langle\psi| & \left(H_{p}-E_{p}\right)\left(H_{p}^{-1}-E_{p}^{-1}\right)|\psi\rangle \\
& =\sum_{K}\left|C_{K}\right|^{2}\left(E_{p K}-E_{p}\right)\left(E_{p K}^{-1}-E_{p}^{-1}\right) \\
& =-\sum_{K}\left|C_{K}\right|^{2}\left(E_{p K}-E_{p}\right)^{2} / E_{p K} E_{p}=0 .
\end{aligned}
$$

Since all elements of the summation are positive, it is valid only when $E_{p}=E_{p K}$ for some $K$ for which $C_{K}$ is nonzero, and $C_{K}=0$ for all other $K$, which means $\psi=C_{K} \Psi_{K}$. So, the sufficiency is also proved. Q.E.D.

The cross- $H$-square equation also has its family. Namely, the following equation:

$$
\left\langle\psi\left|\left(H_{p}-E_{p}\right) H_{p}^{-1}\right| \psi\right\rangle=0
$$

with the definition of $E_{p}$ as

$$
\left\langle\psi\left|H_{p}-E_{p}\right| \psi\right\rangle=0
$$

is necessary and sufficient for the equivalence to the cross$H$-square equation. Also, the following equation,

$$
\left\langle\psi\left|\left(H_{p}^{-1}-{ }^{i} E_{p}\right) H_{p}\right| \psi\right\rangle=0
$$

with the definition of ${ }^{i} E_{p}$ as

$$
\left\langle\psi\left|H_{p}^{-1}-{ }^{i} E_{p}\right| \psi\right\rangle=0
$$

is again necessary and sufficient for the equivalence to the cross- $H$-square equation. The proof of these equations is straightforward and so omitted for brevity. Note that ${ }^{i} E_{p}$ in Eq. (3.39) is not $E_{p}^{-1}$.
When we divide the positive Hamiltonian and its inverse as

$$
\begin{gathered}
H_{p}=\sum_{I}^{N_{D}} e_{I} H_{p I}, \\
H_{p}^{-1}=\sum_{I}^{N_{D}}{ }^{i} e_{I}{ }^{i} H_{p I},
\end{gathered}
$$

then we have cross- $H$-square theorems for the divided Hamiltonians. First, with Eq. (3.40), the following set of equations:

$$
\left\langle\psi\left|\left(H_{p}^{-1}-{ }^{i} E_{p}\right) H_{p I}\right| \psi\right\rangle=0 \quad\left(I=1, \ldots, N_{D}\right)
$$

is equivalent to Eq. (3.39). Second, with Eq. (3.38), the following set of equations,

$$
\left\langle\psi\left|\left(H_{p}-E_{p}\right)^{i} H_{p I}\right| \psi\right\rangle=0 \quad\left(I=1, \ldots, N_{D}\right)
$$

is equivalent to Eq. (3.37). Further, each of the following two sets of equations is equivalent to Eq. (3.35),

$$
\begin{gathered}
\left\langle\psi\left|\left(H_{p}^{-1}-E_{p}^{-1}\right)\left(H_{p I}-E_{p I}\right)\right| \psi\right\rangle=0 \quad\left(I=1, \ldots, N_{D}\right), \\
\left\langle\psi\left|\left(H_{p}-E_{p}\right)\left({ }^{i} H_{p I}-{ }^{i} E_{p I}\right)\right| \psi\right\rangle=0 \quad\left(I=1, \ldots, N_{D}\right),
\end{gathered}
$$

where the partitioned energies $E_{p I}$ and ${ }^{i} E_{p I}$ are defined by

$$
\left\langle\psi\left|H_{p I}-E_{p I}\right| \psi\right\rangle=0, \quad\left\langle\psi\left|{ }^{i} H_{p I}-{ }^{i} E_{p I}\right| \psi\right\rangle=0,
$$

and they satisfy

$$
E_{p}=\sum_{I}^{N_{D}} e_{I} E_{p I}, \quad{ }^{i} E_{p}=\sum_{I}^{N_{D}}{ }^{i} e_{I}{ }^{i} E_{p I} .
$$

The proof of these equations is very similar to that for Eqs. (3.31) and (3.32) and is, therefore, omitted here.

We will see later in the formulation of the generalized ICI theory that these cross- $H$-square equations are important, connecting the two worlds of the regular and inverse Hamiltonians. In this sense, it is natural that this theorem is valid only for the positively shifted Hamiltonian.

The inverse Hamiltonian has the ISE, the inverse variational principle, and the $H^{-1}$-square group of equations, just as the ordinary SE. Therefore, there exists entirely the same theoretical framework as that of the SE on the inverse side of the SE. Further, these two sides are connected by the cross$H$-square equation. The knowledge on the existence of these equivalent sets of equations on both sides of the Hamiltonian would make the physical and chemical imagination more fertile and would help us to understand Nature more clearly.

On the regular side, we usually have an analytical expression of the Hamiltonian, but on the inverse side, we do not necessarily have a closed analytical expression of the Hamiltonian, though we really require it. However, in the matrix form, we can always define the inverse Hamiltonian matrix, 
when we have the regular Hamiltonian matrix. Thus, the concept combining the SE and ISE may have a larger practical merit when we formulate our problem in a matrix representation.

We study in this series of papers [2-6] the structure of the exact wave function. Now that the exact wave function $\psi$ is an eigenfunction of not only $H$ but also $H^{-1}$, the structure of the exact wave function must depend not only on $H$ but also on $H^{-1}$ entirely on the same footing. This fact would lead us to a deeper understanding of the structure of the exact wave function. In the following section, we utilize the concept of the regular and inverse Hamiltonians to generalize the Krylov sequence, and in Sec. V, the ICI theory is generalized into four different ones by connecting the regular and inverse sides of the Hamiltonian.

\section{COMPLETE KRYLOV SEQUENCE}

The Krylov sequence,

$$
\left\{c, \mathbf{A} c, \mathbf{A}^{2} c, \mathbf{A}^{3} c, \ldots\right\},
$$

plays an important role in the eigenvalue problem given by Eq. (2.1) [32,35]. The Arnoldi method [36] and the Lanczos method [37] are related to the Krylov sequence. The Hamiltonian Krylov space

$$
\left\{\psi_{0}, H \psi_{0}, H^{2} \psi_{0}, H^{3} \psi_{0}, \ldots\right\}
$$

is important for solving the SE: these functions are used as basis functions to expand the wave function. As we now know that $\mathbf{A}^{-1}$ plays an equivalent role to $\mathbf{A}$ in the eigenvalue problem, we expect a similar important role of the inverse Krylov sequence defined by

$$
\left\{c, \mathbf{A}^{-1} c, \mathbf{A}^{-2} c, \mathbf{A}^{-3} c, \ldots\right\} .
$$

Similarly, we define the inverse Hamiltonian Krylov sequence as

$$
\left\{\psi_{0}, H^{-1} \psi_{0}, H^{-2} \psi_{0}, H^{-3} \psi_{0}, \ldots\right\} .
$$

Further by combining the two sets of Krylov sequences, we obtain the complete Krylov sequence as

$$
\left\{\ldots, \mathbf{A}^{-3} c, \mathbf{A}^{-2} c, \mathbf{A}^{-1} c, c, \mathbf{A} c, \mathbf{A}^{2} c, \mathbf{A}^{3} c, \ldots\right\}
$$

and the complete Hamiltonian Krylov sequence as

$$
\left\{\ldots, H^{-3} \psi_{0}, H^{-2} \psi_{0}, H^{-1} \psi_{0}, \psi_{0}, H \psi_{0}, H^{2} \psi_{0}, H^{3} \psi_{0}, \ldots\right\} .
$$

The Hamiltonian Krylov sequence gives a basis of the expansion of the exact wave function. Since the ISE is equivalent to the SE, the inverse Krylov sequence should also be important in the expansion of the exact wave function. The complete Krylov sequence given by Eq. (4.6) provides a wider functional space that is useful for calculating the exact wave function.

When we use the Hamiltonian Krylov sequence as a basis of the expansion of a wave function, it sometimes happens that the element of the Krylov subspace does not satisfy the quantum-mechanical $(\mathrm{QM})$ condition a physical wave function must satisfy, i.e., integrable finiteness. For example, when the Hamiltonian includes the Coulombic nuclear attraction operator, it has a singularity at the position of the nucleus, and, therefore, the function $H^{n} \psi_{0}$ with $n \geqslant 1$ does not satisfy the QM condition: $\psi_{0}$ is finite but $H^{n}$ becomes infinite at the nuclear position [see Eq. (6.2), for example] and this nuclear singularity is too large to be solved with the aid of the fact that the nucleus is a finite-size entity [26,27]. This is the difficulty also encountered in the $t$-expansion method [28] and the connected-moments method [29]. Marmorino [30] discussed that this difficulty should also occur in the calculation of the surplus function method [31]. However, we must also note that when $\psi_{0}$ is exact, $H^{n} \psi_{0}$ is simply $E^{n} \psi_{0}$, which implies that a very good reference function is necessary for the convergence of this procedure.

In the present formalism of the Krylov sequence, however, we can use in such a case the inverse Hamiltonian Krylov sequence, where the Coulombic singularity problem does not occur since the elements of the sequence are composed of the inverse of the Hamiltonian, $H^{-n} \psi_{0}$. At the nuclear position, for instance, $H^{-n}$ becomes zero, and at a distance apart from the nuclei, $H^{-n} \psi_{0}$ behaves like $r^{n} \psi_{0}$, which is integrable finite because $\psi_{0}$ decays exponentially like $\sim \exp (-\alpha r)$ or $\sim \exp \left(-\beta r^{2}\right)$. In comparison with the ordinary Krylov sequence, the dependence of this sequence on the quality of the reference function $\psi_{0}$ would be dull and, therefore, the convergence would be faster. In general, it is convenient to use the complete Hamiltonian Krylov sequence given by Eq. (4.6) and use only such parts that satisfy the QM condition. In this case, we can calculate the ground state starting from an approximate excited-state reference function $\psi_{0}$. In conclusion, introducing the inverse of the Hamiltonian, the proposed method is free from the singularity problems originating from the nuclear attraction operator and the electron repulsion operator in the atomic and molecular Hamiltonian given by Eq. (1.1).

\section{GENERALIZED ICI THEORY}

As shown in Sec. III, the ISE has the variational principle and the $H$-square group of equations just as the ordinary SE. Therefore, the theoretical framework developed for the ordinary SE should also be formulated on the inverse side of the SE. Further, the regular and inverse sides are connected by the cross- $H$-square equation, which turns out to be very important and useful in this section. The ICI and ECC theories developed previously for calculating the exact wave function are, therefore, generalized to cover both sides of the Hamiltonian. We describe here the GICI theory. The generalized ECC theory will be given in a forthcoming paper [38]. We show the operator formalism first and then the matrix representation. These formalisms are used in the applications given in Sec. VI.

\section{A. Generalized ICI theory in analytical form}

In Sec. III, we have shown that in order to have a continuous correspondence between the eigenvalues of the regu- 
lar and the inverse Hamiltonian, we have to introduce the positive Hamiltonian, which is easily obtained by shifting the origin of the Hamiltonian as in Eq. (3.16). For the positive Hamiltonian, we have the inverse Ritz variational principle and the cross- $H$-square equation, but for nonpositive Hamiltonian, these useful relations do not hold. Therefore, in this section, we limit ourselves to use only the positive Hamiltonian. Though we do not use the subscript $p$ like in $H_{p}$, for simplicity, all the quantities given in this section are those of the positive Hamiltonian.

The generalized ICI theory will be grouped into four, depending on how the $S$ operator is defined and what variational principle is used in calculating the unknown variables in the $S$ operator. Depending on whether the regular Hamiltonian or the inverse Hamiltonian is used, we denote $R$ or $I$, respectively, so that $R-R, R-I, I-R$, and $I-I$ cases occur. We explain these four cases below.

First are the $R-R$ and $R-I$ cases, where we start from the division of the regular Hamiltonian

$$
H=\sum_{I}^{N_{D}} d_{I} H_{I} .
$$

We note again that we have assigned the coefficients $\left\{d_{I}\right\}$ in front of the divided Hamiltonian $H_{I}$, differently from the partitioning of the Hamiltonian given in Paper II. These coefficients are introduced just for making it clear that all we need to know is the functional form of $H_{I}$ and we need not know the coefficients $d_{I}$. In other words, this coefficient $d_{I}$ was assumed to be unity in the previous formalism [2-4]. Corresponding to Eq. (5.1), we define the variable operator $S$ by

$$
S=\sum_{I=1}^{N_{D}} C_{I} H_{I} .
$$

We here note that in the definition of the $S$ operator, we do not use the coefficients $\left\{d_{i}\right\}$ in Eq. (5.1): we need not know the coefficients $\left\{d_{I}\right\}$. The ICI method is defined by the recurrence

$$
\psi_{n}=\left(1+S_{n}\right) \psi_{n-1},
$$

where $n$ denotes iteration number.

Now, to calculate the unknown coefficients $\left\{C_{I}\right\}$ in the $S$ operator, we can use either the regular or the inverse variational principle. When we use the regular variational principle, it is the $R-R$ case, and we obtain the secular equation

$$
\begin{gathered}
\left\langle\psi_{n}\left|H-E_{n}\right| \psi_{n-1}\right\rangle=0, \\
\left\langle\psi_{n}\left|\left(H-E_{n}\right) H_{I}\right| \psi_{n-1}\right\rangle=0 \quad\left(I=1, \ldots, N_{D}\right) .
\end{gathered}
$$

Note that the energy of the $n$th iteration $E_{n}$ satisfies

$$
\left\langle\psi_{n}\left|H-E_{n}\right| \psi_{n}\right\rangle=0
$$

as well as Eq. (5.4). Because of the variational nature, the energy $E_{n}$ converges to the exact energy from above [6]. At convergence, $\psi_{n}=\psi_{n-1}$, and therefore, Eq. (5.5) is identical to the $H$-square equation given by Eq. (3.9), which guarantees that the solution is exact. This $R-R$ case is the formulation given in Papers I and II.

Next is the $R-I$ case, where we use the inverse variational principle given by Eq. (3.14) to calculate the unknown variables $\left\{C_{I}\right\}$ of the $S$ operator and obtain

$$
\begin{gathered}
\left\langle\psi_{n}\left|H^{-1}-{ }^{i} E_{n}\right| \psi_{n-1}\right\rangle=0, \\
\left\langle\psi_{n}\left|\left(H^{-1}-{ }^{i} E_{n}\right) H_{I}\right| \psi_{n-1}\right\rangle=0 \quad\left(I=1, \ldots, N_{D}\right) .
\end{gathered}
$$

${ }^{i} E_{n}$ also satisfies

$$
\left\langle\psi_{n}\left|H^{-1}-{ }^{i} E_{n}\right| \psi_{n}\right\rangle=0
$$

At convergence, $\psi_{n}=\psi_{n-1}$ and the variational equation given by Eq. (5.8) becomes equal to the cross- $H$-square equation given by Eq. (3.43), which guarantees that the solution is exact. Since the present Hamiltonian is positive, the energy converges from below to the exact inverse energy

In the above formulation, we defined our variable operator $S$ in terms of the regular Hamiltonian. Next, in the $I-R$ and $I-I$ cases, we define our variable operator in terms of the inverse Hamiltonian. For doing so, we first define the division of the inverse Hamiltonian as

$$
H^{-1}=\sum_{I}^{N_{D}} d_{I}{ }^{i} H_{I} .
$$

Again, we have introduced the unimportant coefficients $\left\{d_{l}\right\}$, which we need not know throughout the calculations. For this division of the inverse Hamiltonian, we introduce the variable operator ${ }^{i} S$ as

$$
{ }^{i} S=\sum_{I=1}^{N_{D}}{ }^{i} C_{I}{ }^{i} H_{I}
$$

and define the ICI wave function as

$$
\psi_{n}=\left(1+{ }^{i} S_{n}\right) \psi_{n-1}
$$

Now, we calculate the unknown coefficients $\left\{{ }^{i} C_{l}\right\}$ in the ${ }^{i} S$ operator again either by the regular or the inverse variational principle. The former is the $I-R$ case and the latter is the $I-I$ case. When we use the regular variational principle, we obtain the secular equation for the $I-R$ case,

$$
\begin{gathered}
\left\langle\psi_{n}\left|H-E_{n}\right| \psi_{n-1}\right\rangle=0, \\
\left\langle\psi_{n}\left|\left(H-E_{n}\right)^{i} H_{I}\right| \psi_{n-1}\right\rangle=0 \quad\left(I=1, \ldots, N_{D}\right) .
\end{gathered}
$$

At convergence, this equation is identical to the cross- $H$ square equation given by Eq. (3.44), which guarantees that the solution is exact. In this case, the energy $E_{n}$ converges to the exact energy from above. On the other hand, when we use the inverse variational principle given by Eq. (3.14), we obtain the secular equation for the $I-I$ case,

$$
\left\langle\psi_{n}\left|H^{-1}-{ }^{i} E_{n}\right| \psi_{n-1}\right\rangle=0
$$




$$
\left\langle\psi_{n}\left|\left(H^{-1}-{ }^{i} E_{n}\right)^{i} H_{I}\right| \psi_{n-1}\right\rangle=0 \quad\left(I=1, \ldots, N_{D}\right) .
$$

At convergence, this is identical to the $H^{-1}$-square equation given by Eq. (3.32), so that the converged solution is exact. In the $I-I$ case, the energy converges from below to the exact inverse energy.

Thus, from the SE and ISE, we have four different ICI methods for calculating the exact wave function. One would converge more quickly than others and one would be more easily formulated than others. A criterion for the choice is the existence of singularities in the Hamiltonian or in the inverse Hamiltonian. For atomic and molecular systems, the largest singularity is due to the nuclear attraction operator. In this case, the integrals involving higher products of the nuclear attraction operator may diverge and do not satisfy the QM condition necessary for physical wave functions, so that the calculation is problematic. Such a situation may occur when we use the $S$ operator defined by using the divided regular Hamiltonian, Eq. (5.1). Namely, the $R-R$ and $R-I$ cases are problematic. Therefore, we recommend using the ${ }^{i} S$ operator defined by Eq. (5.11): the variational principle may be either regular or inverse, which is the $I-R$ or $I-I$ case.

It is convenient if we can combine two different definitions of the recurrence formula of the ICI theory, Eqs. (5.3) and (5.12), into one. This is possible by introducing the combined recurrence formula as

$$
\psi_{n}=\left(1+S_{n}+{ }^{i} S_{n}\right) \psi_{n-1} .
$$

In using this formula, a remark is necessary. When the QM condition is not satisfied by the elements of $S_{n}$ and/or ${ }^{i} S_{n}$, we must omit them from the calculations. When we start from an approximate higher excited state as an initial guess to calculate the ground state, some deexcitations are done by $S_{n}$ or ${ }^{i} S_{n}$ and, therefore, we need the terms belonging to both $S_{n}$ and ${ }^{i} S_{n}$. For the ordinary case, however, using Eq. (5.17) is essentially the same as using Eq. (5.3) or Eq. (5.12) separately.

\section{B. Generalized ICI theory in matrix representation}

In the above operator formalism of the ICI theory, we need an explicit form of the inverse Hamiltonian in the $R-I$, $I-R$, and $I-I$ cases, but it is not necessarily given in a closed analytical form. However, in the matrix formulation of quantum mechanics, we can always define the inverse of the Hamiltonian, so that the matrix representation of the ICI theory has certainly a merit in actual calculations.

Suppose that we have a set of orthonormal configuration functions $\left\{\boldsymbol{\chi}_{i}\right\}$ for the system under consideration. It may be a complete configuration space of full CI made of some orthonormal set of orbitals such as Hartree-Fock orbitals, or some subspace of this set, such as the singles to quadruples (SDTQ) configuration space. We define the Hamiltonian matrix defined in these configuration functions as

$$
\mathbf{H}=\left\{H_{i j}\right\}=\left\{\left\langle\chi_{i}|H| \chi_{j}\right\rangle\right\} .
$$

For convenience, we assume in this section that we have shifted our Hamiltonian $H$ to be positive as defined by Eq. (3.16), though we do not use the subscript $p$ for simplicity. The matrix $\mathbf{H}$ is then positive definite. The eigenvalue problem of this Hamiltonian is given by

$$
\mathbf{H} \boldsymbol{\lambda}=E \boldsymbol{\lambda},
$$

and that of the inverse Hamiltonian $\mathbf{H}^{-1}$ is given by

$$
\mathbf{H}^{-1} \boldsymbol{\lambda}=E^{-1} \boldsymbol{\lambda},
$$

where $\boldsymbol{\lambda}$ is an eigenvector and $E$ and $E^{-1}$, which are positive, are the eigenvalue and its inverse. The corresponding wave function is written as

$$
\psi=\chi \lambda=\sum_{i} \lambda_{i} \chi_{i}
$$

where $\boldsymbol{\chi}$ is a row vector and $\boldsymbol{\lambda}$ is a column vector. When $\boldsymbol{\chi}$ involves full configurations, as we assume below, this is nothing else but the full CI, and when $\boldsymbol{\chi}$ involves only SDTQ functions, it is SDTQ-CI.

In the matrix representation of ICI, we have four different cases, $R-R, R-I, I-R$, and $I-I$, as in the operator formalism. In the $R-R$ and $R-I$ cases, we first divide the $\mathbf{H}$ matrix as in Eq. (5.1),

$$
\mathbf{H}=\sum_{I}^{N_{D}} d_{I} \mathbf{H}_{I}
$$

and define the $\mathbf{S}$ operator as

$$
\mathbf{S}=\sum_{I=1}^{N_{D}} C_{I} \mathbf{H}_{I}
$$

Then, the ICI is defined by the recurrence

$$
\boldsymbol{\lambda}_{n}=\left(\mathbf{1}+\mathbf{S}_{n}\right) \boldsymbol{\lambda}_{n-1},
$$

where $n$ is the iteration number. For the formulations below, it is convenient to introduce the coefficient $C_{0}$ for the unit matrix $\mathbf{1}$ of the above ICI equation, and redefine the ICI as

$$
\boldsymbol{\lambda}_{n}=\left(\sum_{l=0}^{N_{D}} C_{I, n} \mathbf{H}_{I}\right) \boldsymbol{\lambda}_{n-1},
$$

where $\mathbf{H}_{0}=\mathbf{1}$.

In the $R-R$ case, we apply the regular variational principle to this expression and obtain the secular equation of the dimension $N_{D}+1$,

$$
\left(\mathbf{h}_{n}-E_{n} \mathbf{s}_{n}\right) \mathbf{C}_{n}=0,
$$

where $\mathbf{C}_{n}$ is a column vector composed of the coefficients $C_{I, n}$ and the matrix elements of $\mathbf{h}_{n}$ and $\mathbf{s}_{n}$ are given by

$$
\left(\mathbf{h}_{n}\right)_{I J}=\boldsymbol{\lambda}_{n-1}^{*} \mathbf{H}_{I}^{*} \mathbf{H H}_{J} \boldsymbol{\lambda}_{n-1}
$$


and

$$
\left(\mathbf{s}_{n}\right)_{I J}=\boldsymbol{\lambda}_{n-1}^{*} \mathbf{H}_{I}^{*} \mathbf{H}_{J} \boldsymbol{\lambda}_{n-1} .
$$

As we perform iterative calculations, the energy decreases, and at convergence the eigenvalue $E_{n}$ and the eigenvector $\boldsymbol{\lambda}_{n}$ become identical with the solution of the full CI given by Eq. (5.19).

In the $R-I$ case, we use the inverse variational principle. By applying it to the ICI recurrence formula given by Eq. (5.25), we obtain the secular equation

$$
\left(\mathbf{h}_{n}^{-1}-{ }^{i} E_{n} \mathbf{s}_{n}\right) \mathbf{C}_{n}=0
$$

where $\mathbf{s}_{n}$ is the same as that given by Eq. (5.28) and $\mathbf{h}_{n}^{-1}$ is given by

$$
\left(\mathbf{h}_{n}^{-1}\right)_{I J}=\boldsymbol{\lambda}_{n-1}^{*} \mathbf{H}_{I}^{*} \mathbf{H}^{-1} \mathbf{H}_{J} \boldsymbol{\lambda}_{n-1},
$$

where $\mathbf{H}^{-1}$ is the inverse of the Hamiltonian matrix. We perform iterative calculations of the small eigenvalue problem given by Eq. (5.29). The energy approaches from below the inverse of the full-CI energy and at convergence, we obtain the inverse energy and the eigenvector that are identical to the full-CI values.

In the above formulation, the variable operator $\mathbf{S}$ of the ICI was defined by using the regular Hamiltonian. In the $I-R$ and $I-I$ cases, we define it using the inverse Hamiltonian. First, we define a partition of the inverse Hamiltonian,

$$
\mathbf{H}^{-1}=\sum_{I}^{N_{D}} d_{I}{ }^{i} \mathbf{H}_{I},
$$

and define the ${ }^{i} \mathbf{S}$ operator as

$$
{ }^{i} \mathbf{S}=\sum_{I=1}^{N_{D}}{ }^{i} C_{I}{ }^{i} \mathbf{H}_{I} .
$$

Then, the ICI is given by the recurrence

$$
\boldsymbol{\lambda}_{n}=\left(\mathbf{1}+{ }^{i} \mathbf{S}_{n}\right) \boldsymbol{\lambda}_{n-1} .
$$

For convenience, we assign the coefficient ${ }^{i} C_{0}$ for the unit matrix 1 of the above ICI equation, and redefine the ICI as

$$
\boldsymbol{\lambda}_{n}=\left(\sum_{I=0}^{N_{D}}{ }^{i} C_{I, n}{ }^{i} \mathbf{H}_{I}\right) \boldsymbol{\lambda}_{n-1},
$$

where ${ }^{i} \mathbf{H}_{0}=\mathbf{1}$.

We apply first the regular variational principle to the ICI wave function given by Eq. (5.34) and obtain the secular equation for the $I-R$ case as

$$
\left({ }^{i} \mathbf{h}_{n}-E_{n}{ }^{i} \mathbf{s}_{n}\right) \mathbf{C}_{n}=0
$$

where the matrix elements of ${ }^{i} \mathbf{h}_{n}$ and ${ }^{i} \mathbf{s}_{n}$ are given by

$$
\left({ }^{i} \mathbf{h}_{n}\right)_{I J}=\boldsymbol{\lambda}_{n-1}^{*}{ }^{i} \mathbf{H}_{I}^{*} \mathbf{H}^{i} \mathbf{H}_{J} \boldsymbol{\lambda}_{n-1}
$$

and

$$
\left({ }^{i} \mathbf{S}_{n}\right)_{I J}=\boldsymbol{\lambda}_{n-1}^{*}{ }^{i} \mathbf{H}_{I}^{* i} \mathbf{H}_{J} \boldsymbol{\lambda}_{n-1} .
$$

In iterations, the energy approaches from above the full-CI energy.

Next, in the I-I case, we apply the inverse variational principle to the ICI recurrence given by Eq. (5.34) and obtain the secular equation of the dimension $N_{D}+1$ as

$$
\left({ }^{i} \mathbf{h}_{n}^{-1}-{ }^{i} E_{n}{ }^{i} \mathbf{s}_{n}\right) \mathbf{C}_{n}=0
$$

where the matrix ${ }^{i} \mathbf{h}_{n}^{-1}$ is defined by

$$
\left({ }^{i} \mathbf{h}_{n}^{-1}\right)_{I J}=\boldsymbol{\lambda}_{n-1}^{*}{ }^{i} \mathbf{H}_{I}^{*} \mathbf{H}^{-1 i} \mathbf{H}_{J} \boldsymbol{\lambda}_{n-1},
$$

and the overlap matrix ${ }^{i} \mathbf{S}_{n}$ is common to that given by Eq. (5.37). After iteration, the solution of Eq. (5.38) should approach from below the inverse of the full-CI energy.

In the above formulation, we used the matrices $\mathbf{H}, \mathbf{H}_{I}$, etc. of the full-CI dimension, since we adopted the complete basis functions $\left\{\chi_{i}\right\}$ for our matrix representation. In actual ICI calculations, it is unnecessary to keep such huge matrices in memory. On the other hand, the matrices that appear in the ICI secular equations, such as $\mathbf{h}_{n}$ and $\mathbf{s}_{n}$ of Eq. (5.26), have only $N_{D}+1$ dimension. Actually, the present matrix representation is equivalent to calculating the necessary integrals by the resolution of identity method using the complete basis $\left\{\chi_{i}\right\}[6]$.

In the matrix formulation, the singularity problem is not so explicit, because all the individual matrices are well defined. For a small basis function space, no problem would occur except that the convergence would be slower in the problematic case. But, as the basis function space approaches completeness, the product of the matrices approaches the matrix of the operator product, so that the same problem as in the analytical procedure would occur in the problematic case even in the matrix formulation. We see below such an example in the application to hydrogen atom.

\section{GENERALIZED ICI THEORY APPLIED TO HYDROGEN ATOM}

The hydrogen atom is the simplest basic system that shows the importance of the ISE concept, because the regular Hamiltonian has a singularity due to the nuclear attraction operator. The ordinary ICI theory (GICI in the $R-R$ case) is problematic for this singularity, but we show that the generalized ICI theory in the $I-R$ and $I-I$ cases has no problem. The ECC study will be given in the forthcoming paper [38].

The Hamiltonian for the radial part with zero angular momentum is written as

$$
H=k+\nu=-\frac{1}{2} \frac{d^{2}}{d r^{2}}-\frac{1}{r} \frac{d}{d r}-\frac{1}{r},
$$

where the first two terms represent the kinetic operator $k$ and the last term represents the nuclear attraction potential $\nu$. This Hamiltonian has a singularity at the origin in the last two terms, so that the higher elements of the ordinary Krylov sequence have a strong singularity at the origin and do not 
satisfy the QM condition. For example, the following integral diverges even for the exact wave function $\psi=\exp (-r)$ [30]:

$$
\langle\psi|(-1 / r) H(-1 / r)| \psi\rangle=-\infty,
$$

but the next one does not,

$$
\langle\psi|(-r) H(-r)| \psi\rangle=-1.0 .
$$
as

We define the positive Hamiltonian by shifting the origin

$$
H_{p}=H+1 .
$$

We show here the application of the generalized ICI theory to the hydrogen atom first in the analytical form and then in the matrix representation.

\section{A. Analytical application of the generalized ICI theory}

The ordinary ICI method, which is the generalized ICI in the $R-R$ case, is problematic, because of the Coulombic singularity due to the nuclear attraction operator. Some integrals involved are divergent, just like the integral shown in Eq. (6.2). Therefore, the ICI in the $R-R$ case is not applicable, in its analytical form, even to the hydrogen atom. This problem is circumvented by using the generalized ICI theory based on the ISE concept, in particular, the cross- $H$-square theorem. We show below the use of the generalized ICI theory in the $I-R$ case.

To utilize the ISE concept, we need an explicit expression of the inverse Hamiltonian. Different from the matrix formulation, this is not necessarily straightforward in the analytic treatment. But, we may write the inverse of the Hamiltonian given by Eq. (6.1) as

$$
\begin{aligned}
H^{-1} & =\frac{1}{k+\nu}=\frac{1}{\nu}\left(1+\frac{1}{\nu} k\right)^{-1} \\
& =\frac{1}{\nu}\left\{1-\frac{1}{\nu} k+\left(\frac{1}{\nu} k\right)^{2}-\left(\frac{1}{\nu} k\right)^{3}+\cdots\right\} .
\end{aligned}
$$

In Eq. (6.5b), the kinetic operator is not in the inverse form. Putting $1 / \nu=-r$, we see no singularity in the inverse Hamiltonian. The inverse of the positive Hamiltonian given by Eq. (6.4) is written similarly as

$$
\begin{aligned}
H_{p}^{-1} & =\frac{1}{k+\nu+1}=\frac{1}{\nu}\left(1+\frac{k+1}{\nu}\right)^{-1} \\
& =\sum_{I=1} \frac{1}{\nu}\left(-\frac{k+1}{\nu}\right)^{I-1} .
\end{aligned}
$$

The generalized ICI theory is defined by using the positive Hamiltonian given by Eqs. (6.4) and (6.6). Based on the expression of the inverse positive Hamiltonian given by Eq. (6.6b), we define the variable operator ${ }^{i} S$ as in Eq. (5.11),
TABLE I. Energy of the hydrogen atom calculated by the $I-R$ type ICI method with increasing accuracy of the ${ }^{i} S$ operator.

\begin{tabular}{ccl}
\hline \hline${ }^{i} S^{\mathrm{a}}$ & $n^{\mathrm{b}}$ & \multicolumn{1}{c}{$E^{\mathrm{c}}$} \\
\hline${ }^{i} S$ of Eq. (6.7) & & \\
$\psi_{0}$ & 4 & 0.625 \\
${ }^{i} S(1)$ & 3 & 0.5070544 \\
${ }^{i} S(2)$ & 3 & 0.5005974 \\
${ }^{i} S(3)$ & 3 & 0.5000426 \\
${ }^{i} S(4)$ & 2 & 0.5000026 \\
${ }^{i} S(5)$ & 1 & 0.5000001 \\
${ }^{i} S(6)$ & & 0.5000000 \\
${ }^{i} S$ of Eq. $(6.10)$ & & \\
$\psi_{0}$ & 4 & 0.625 \\
${ }^{i} S(1)$ & 3 & 0.5070544 \\
${ }^{i} S(2)$ & 2 & 0.5004132 \\
${ }^{i} S(3)$ & 2 & 0.5000207 \\
${ }^{i} S(4)$ & 2 & 0.5000011 \\
${ }^{i} S(5)$ & 1 & 0.5000001 \\
${ }^{i} S(6)$ & & 0.5000000 \\
\hline \hline
\end{tabular}

${ }^{\text {a }}$ Truncated ${ }^{i} S$ operator.

${ }^{b}$ Number of iterations at which the energy converges by seven decimal figures.

${ }^{c}$ Positively shifted by +1.0 . The exact energy is 0.5 a.u. for the positive Hamiltonian and -0.5 a.u. for the nonpositive Hamiltonian.

$$
{ }^{i} S=\sum_{I=1}^{\infty} C_{I} \frac{1}{\nu}\left(-\frac{k+1}{\nu}\right)^{I-1} .
$$

In this case, the number of divisions $N_{D}$ is infinity. Using this operator, the generalized ICI wave function is defined by the recurrence

$$
\psi_{n}=\left(1+{ }^{i} S_{n}\right) \psi_{n-1},
$$

where $n$ is the iteration number.

We calculate the ICI wave function by successively truncating the terms of Eq. (6.7): first ${ }^{i} S(1)=C_{1}(1 / \nu)$, second ${ }^{i} S(2)=C_{1}(1 / \nu)-C_{2}\left(1 / \nu^{2}\right)(k+1)$, third ${ }^{i} S(3)=C_{1}(1 / \nu)$ $-C_{2}(1 / \nu)[(k+1) / \nu]+C_{3}(1 / \nu)[(k+1) / \nu][(k+1) / \nu]$, and so on. The initial guess is the Slater-type function

$$
\psi_{0}=\exp (-\alpha r),
$$

with $\alpha=1.5$ for which the energy expectation value is 0.625 a.u. with the positive Hamiltonian $(-0.375$ a.u. with the regular Hamiltonian). $\alpha=1.0$ is the exact wave function with $E_{p}=0.5$ a.u. for $H_{p}(E=-0.5$ a.u. for the regular $H)$. We optimize the unknown variables $C_{i}$ by the regular variational method for the shifted positive Hamiltonian. This is the GICI in the $I-R$ case and the basic secular equation, which is given by Eqs. (5.13) and (5.14), becomes the cross- $H$-square equation at convergence.

Table I gives the overall result and the convergence process is shown in Table II for ${ }^{i} S(1),{ }^{i} S(3)$, and ${ }^{i} S(5)$. When we use only ${ }^{i} S(1)$, the ICI converges by four iterations: the 
TABLE II. Convergence process for each ${ }^{i} S(I)(I=1,3,5)$ of Eq. (6.7).

\begin{tabular}{ccl}
\hline \hline${ }^{i} S$ & Iteration & \multicolumn{1}{c}{ Energy $^{\mathrm{a}}$} \\
\hline${ }^{i} S(1)$ & 0 & 0.625 \\
& 1 & 0.5089746 \\
& 2 & 0.5071126 \\
& 3 & 0.5070563 \\
${ }^{i} S(3)$ & 4 & 0.5070544 \\
& 0 & 0.625 \\
& 1 & 0.5000459 \\
${ }^{i} S(5)$ & 2 & 0.5000427 \\
& 3 & 0.5000426 \\
& 0 & 0.625 \\
& 1 & 0.5000002 \\
& 2 & 0.5000001 \\
\hline \hline
\end{tabular}

The variable coefficient $C_{I}$ converges toward zero.

coefficient $C_{1}$ converges toward 0.0 and the converged energy is 0.507054 a.u. which is higher than the exact value, 0.5 a.u. This is because ${ }^{i} S(1)$ is only a part of the inverse Hamiltonian. For obtaining the exact solution, the ${ }^{i} S$ operator must include all parts of the divided inverse Hamiltonian. When we use ${ }^{i} S(2)$, the converged energy is 0.500597 , becoming closer to 0.5 . For ${ }^{i} S(3)$, the convergence is very quick as shown in Table II and the converged energy 0.500043 is closer to 0.5 . With ${ }^{i} S(5)$, the converged energy becomes much closer to 0.5 , and with ${ }^{i} S(6)$, the energy converges to the exact value within seven decimal figures. The convergence is good not only for the individual level of ${ }^{i} S(I)$, but also for the overall level. Though the ${ }^{i} S$ operator is composed of infinite number of terms in the expression given by Eq. (6.7), only the first six terms are enough to get the energy correct to seven decimal figures.

In the expansion of the ${ }^{i} S$ operator given by Eq. (6.7), the operator $(k+1)$ can be omitted, and we can expand the ${ }^{i} S$ operator solely by the inverse potential $-r$,

$$
{ }^{i} S=\sum_{l=1}^{\infty} C_{I}\left(\frac{1}{\nu}\right)^{I} .
$$

This is possible because the operator $(k+1)$ in Eq. (6.7) gives the terms in lower orders of $r$, which already exist in the individual ${ }^{i} S(I)$ term. In other words, each ${ }^{i} S(I)$ term of Eq. (6.7) can be rearranged in the form of the ${ }^{i} S(I)$ term of Eq. (6.10): no new order terms appear in Eq. (6.7) in comparison with Eq. (6.10). Further, we can look at Eq. (6.10) as an expansion of the ${ }^{i} S$ operator by the basic variable $r$ of this system: for the $S$ state of the hydrogen atom, the basic variable is only one that is $r$ and, therefore, the ${ }^{i} S$ operator should be able to be expanded by this variable as given by Eq. (6.10).

In Table I we also give the converged energy for each choice of ${ }^{i} S(I)$ of Eq. (6.10) from $I=1$ to $I=6$. The initial energies by the ${ }^{i} S(I)$ of Eqs. (6.7) and (6.10) were completely the same and the converged energies were also close to each other.
We emphasize here that no problem of the Coulombic singularity has occurred in the above calculations of the $I-R$ case of the GICI theory, in contrast to the case of using the ordinary Hamiltonian operator in the ordinary ICI method, which is the $R-R$ case of the GICI theory. This would also have been the case in the $t$-expansion method [28] and the connected-moments method [29]. Thus, the use of the ISE concept given in this paper would overcome a well-known long-continued difficulty of singularities in the study of accurate wave functions.

\section{B. Application of the generalized ICI theory in matrix representation}

We show here the usage of the present concept of the SE and the ISE by applying the matrix representation of the generalized ICI theory to the hydrogen atom. This application is particularly interesting because we can show all four cases of the generalized ICI theory. Different from the analytical case given above, we have an explicit form of the inverse Hamiltonian matrix without ambiguity.

The basis functions of the matrix representation are the STO- $N G$ sets $(N=4$ and 8$)$ of $O$-ohata, Taketa, and Huzinaga [39]. For the STO- $8 G$ orbital, we further added two GTO's of very large exponents, 2000 and 10000 , to reinforce the region close to the nucleus. The orthonormal basis orbitals $\left\{\chi_{i}\right\}$ are made by diagonalizing the overlap matrix and transforming the basis orbitals in terms of the Löwdin orthogonalization [40]. By diagonalizing the positive Hamiltonian matrix defined by Eq. (6.4), we obtain the energy of the ground state as 0.501009 a.u. and 0.500004 a.u., respectively, by the STO- $4 G$ and STO- $(8+2) G$ sets. The corresponding inverse energy is 1.995970 a.u. and 1.999985 a.u., respectively. Thus, these energies and inverse energies are our goal in the matrix representation GICI calculations.

In doing the GICI, the Hamiltonian and the inverse Hamiltonian are divided as

$$
\mathbf{H}=\mathbf{v}+(\mathbf{k}+\mathbf{1})
$$

and

$$
\mathbf{H}^{-1}=\mathbf{v}^{-1}+\mathbf{r}
$$

respectively, where $\mathbf{v}$ is the matrix of the potential operator, $\mathbf{k}$ the matrix of the kinetic operator, and $\mathbf{r}$ is the residual matrix. The $S$ and ${ }^{i} S$ operators are defined, corresponding to the above equations, as

$$
\mathbf{S}=C_{\nu} \mathbf{v}+C_{k}(\mathbf{k}+\mathbf{1})
$$

and

$$
{ }^{i} \mathbf{S}={ }^{i} C_{\nu} \mathbf{v}^{-1}+{ }^{i} C_{r} \mathbf{r},
$$

respectively.

Table III shows the converging process of the four types of the generalized ICI calculations of the hydrogen atom based on the STO- $4 G$ basis set. The initial reference function $\psi_{0}$ is the second outermost Löwdin orthogonalized orbital: the first one was too good as the initial guess to show 
TABLE III. Generalized ICI calculations in matrix form for the hydrogen atom using a positive Hamiltonian for the STO- $4 G$ basis set. The correct energy for the positive Hamiltonian of the STO- $4 G$ basis is $E_{p}=0.501009$ a.u. and $E_{p}^{-1}=1.995970$ a.u. $^{-1}$.

\begin{tabular}{ccccc}
\hline \hline & \multicolumn{4}{c}{ Energy } \\
\cline { 2 - 5 } Interation & $R-R$ case & $I-R$ case & $R-I$ case & $I-I$ case \\
\hline 1 & 0.554112 & 0.827107 & 1.909055 & 1.758407 \\
2 & 0.522819 & 0.502693 & 1.949066 & 1.995881 \\
3 & 0.507856 & 0.501013 & 1.975771 & 1.995969 \\
4 & 0.503171 & 0.501009 & 1.987305 & 1.995970 \\
5 & 0.501761 & & 1.991964 & \\
6 & 0.501103 & & 1.994120 & \\
7 & 0.501043 & & 1.995084 & \\
8 & 0.501022 & & 1.995546 & \\
9 & 0.501014 & & 1.995763 & \\
10 & 0.501011 & & 1.995869 & \\
11 & 0.501010 & & 1.995921 & \\
12 & 0.501010 & & 1.995946 & \\
13 & 0.501010 & & 1.995958 & \\
14 & 0.501009 & & 1.995964 & \\
15 & & & 1.995967 & \\
16 & & & 1.995969 & \\
17 & & & 1.995969 & \\
18 & & & 1.995970 & \\
\hline \hline
\end{tabular}

the converging process. With the rather crude STO- $4 G$ basis, all four types of the generalized ICI calculations have converged. The $R-R$ and $I-R$ cases converge to $E_{p}$ $=0.501009$ a.u. and the $R-I$ and $I-I$ cases to $E_{p}^{-1}$ $=1.995970$ a.u. In the analytical case, the $R-R$ and $R-I$ cases involve the calculations of the diverging integrals such as that expressed by Eq. (6.2). However, in the matrix representation with the crude STO- $4 G$ basis set, even these problematic cases showed no difficulty in the convergence process. On the other hand, the $I-R$ and $I-I$ cases that have no intrinsic theoretical problem show remarkably good convergence: they converge with only four iterations, in contrast to 14 and 18 for the $R-R$ and $R-I$ cases. In the $R-R$ and $I-R$ cases, which use the normal variational principle, the energy converges from above, and in the $R-I$ and $I-I$ cases, which use the inverse variational principle, the energy converges monotonically from below to the correct value.

We next show the GICI calculations for the STO- $(8$ $+2) G$ basis set. The STO- $8 G$ basis is already a much better basis than the STO- $4 G$ basis. The energy of the former is 0.500009 a.u. in comparison with that of the latter, 0.501009 a.u. We further added two large exponent bases in order to reinforce the inner region of the $\mathrm{AO}$ where the divergence of the integrals such as that given by Eq. (6.2) is dictated. By this addition, the energy decreases slightly to 0.500004 a.u. The initial reference function $\psi_{0}$ is the outermost Löwdin orthogonalized orbital.

For this better basis, the convergences of the $R-R$ and $R-I$ cases become much worse than for the STO- $4 G$ basis: actually, the convergence was too slow to be realistic. This is
TABLE IV. Generalized ICI calculations in matrix form for the hydrogen atom using the positive Hamiltonian for the STO- $(8$ $+2) G$ basis set. The correct energy for the positive Hamiltonian of the STO- $(8+2) G$ basis is $E_{p}=0.500004$ a.u. and $E_{p}^{-1}$ $=1.999985$ a.u. $^{-1}$. For the $R-R$ and $R-I$ cases, the convergence was too slow to be realistic.

\begin{tabular}{cccccc}
\hline \hline & \multicolumn{2}{c}{$I-R$ case } & & \multicolumn{2}{c}{$I-I$ case } \\
\cline { 2 - 3 } \cline { 5 - 6 } Iteration & Guess $1^{\mathrm{a}}$ & Guess 2 & & Guess $1^{\mathrm{a}}$ & Guess 2 \\
\hline 1 & 0.505034 & 0.973440 & & 1.960160 & 1.930675 \\
2 & 0.500161 & 0.502669 & & 1.999802 & 1.998416 \\
3 & 0.500013 & 0.500287 & & 1.999972 & 1.999825 \\
4 & 0.500005 & 0.500024 & & 1.999984 & 1.999971 \\
5 & 0.500004 & 0.500005 & & 1.999985 & 1.999984 \\
6 & & 0.500004 & & 1.999985 \\
\hline
\end{tabular}

${ }^{\mathrm{a}}$ The outermost orthogonalized orbital is the initial guess function $\psi_{0}$.

${ }^{\mathrm{b}}$ The second outermost orthogonalized orbital is the initial guess function $\psi_{0}$.

expected since these cases are very problematic in the analytical case because of the existence of the diverging integrals. As the basis set is improved, the behavior of the matrix formulation would become closer to the analytical one. Thus, Table IV shows only the $I-R$ and $I-I$ cases. For these cases, however, the convergence was quite fantastic: the energy converges only with five iterations in both cases. This may be expected, since there is no intrinsic theoretical problem in these cases, but the performance is much better than expected. Since the convergence was so beautiful for the $I-R$ and $I-I$ cases, we tried the calculations using a worse initial guess, which is the second outermost orthogonalized orbital. Again, both of the $I-R$ and $I-I$ cases showed quite a nice convergence with only six iterations. For the $I-R$ case, it converges monotonically from above to the correct value, and for the $I-I$ case, it converges from below to the correct value.

It is interesting to see the effect of using the positive Hamiltonian. It has been shown in the previous section that without introducing the positive Hamiltonian, a natural connection between the regular and the inverse worlds is difficult and we cannot obtain the cross- $H$-square equations, so that the formulation of the $I-R$ and $R-I$ cases becomes impossible.

Table V shows the generalized ICI calculations for the STO- $4 G$ basis set using a nonpositive Hamiltonian, whose energy eigenvalue is -0.498990 a.u. and -2.004046 a.u. for the inverse case. The problematic $R-R$ and $R-I$ cases show a behavior similar to that of Table III. The $I-R$ case, which has no theoretical problem with the positive Hamiltonian, shows some problem with the nonpositive Hamiltonian, because the cross- $H$-square theorem does not hold in this case. Namely, the convergence becomes much worse when we use the nonpositive Hamiltonian (13 times in comparison with only five times). The $I-I$ case shows a good convergence (only four iterations) and is insensitive to whether the Hamiltonian is positive or not, as seen from the fact that the 
TABLE V. Generalized ICI calculations in matrix form with the nonpositive Hamiltonian for the hydrogen atom with the STO- $4 G$ basis set. The correct energy for the STO- $4 G$ basis is $E=-0.498990$ a.u. and $E^{-1}=-2.004046$ a.u. $^{-1}$.

\begin{tabular}{ccccc}
\hline \hline & \multicolumn{4}{c}{ Energy } \\
\cline { 2 - 5 } Iteration & $R-R$ case & $I-R$ case & $R-I$ case & $I-I$ case \\
\hline 1 & -0.445888 & -0.436302 & -1.588918 & -1.965856 \\
2 & -0.477181 & -0.496044 & -1.834904 & -2.003510 \\
3 & -0.492144 & -0.498222 & -1.940275 & -2.004033 \\
4 & -0.496829 & -0.498678 & -1.980685 & -2.004046 \\
5 & -0.498239 & -0.498842 & -1.995473 & \\
6 & -0.498729 & -0.498920 & -2.000914 & \\
7 & -0.498897 & -0.498956 & -2.002900 & \\
8 & -0.498957 & -0.498973 & -2.003627 & \\
9 & -0.498978 & -0.498982 & -2.003893 & \\
10 & -0.498986 & -0.498986 & -2.003990 & \\
11 & -0.498989 & -0.498988 & -2.004025 & \\
12 & -0.498990 & -0.498989 & -2.004039 & \\
13 & & -0.498990 & -2.004043 & \\
14 & & & -2.004045 & \\
15 & & & -2.004046 & \\
\hline \hline
\end{tabular}

$H^{-1}$-square equation is valid for both nonpositive and positive Hamiltonians. It is noteworthy that both the $R-I$ and $I-I$ cases of the nonpositive Hamiltonian converge from above to the correct value, as the ordinary variational case, contrary to the behavior from below in Table III, which is theoretically correct for the positive Hamiltonian.

Table VI shows a similar convergence process for the nonpositive Hamiltonian with STO- $(8+2) G$ basis. In com- parison with Table IV, the effect of taking a positive Hamiltonian is remarkable for the $I-R$ case. In the nonpositive Hamiltonian case, which is theoretically bad in the $I-R$ case, the convergence to the correct value, -0.499996 a.u., takes 50 iterations in contrast to only five (guess 1 ) shown in Table IV for the positive Hamiltonian case. This is a very natural consequence, since the calculation must be done as the theory requires. Even in the $I-I$ case, the convergence for the positive Hamiltonian is better than the nonpositive case. With the nonpositive Hamiltonian, the $I-I$ case gives the convergence from above, different than "from below" seen for the positive Hamiltonian given in Table IV.

\section{CONCLUSION}

Introducing the inverse of the Hamiltonian, we obtain the inverse Schrödinger equation (ISE) that is entirely equivalent to the ordinary Schrödinger equation (SE). The ISE has the same determinative power as the regular SE. The inverse forms of the variational principle and the $H$-square group of equations that are equivalent to the ISE are derived, and by further shifting our Hamiltonian to be positive, the inverse Ritz variational principle and the cross- $H$-square equations are derived. For the positive Hamiltonian, we obtain a monotonic correspondence between the energy eigenvalues of the regular and inverse Hamiltonians. In the variational process, the inverse energy approaches "from below" the exact energy, in contrast to "from above" in the ordinary case. The knowledge on the equivalent sets of equations on the regular and inverse sides of the Hamiltonian, including the SE and ISE, not only makes the imaginations on physical and chemical phenomena fertile and gives a deep insight on the nature of the quantum-mechanical entities, but also provides an ef-

TABLE VI. Generalized ICI calculations in matrix form with the nonpositive Hamiltonian for the hydrogen atom with the STO- $(8+2) G$ basis set. The correct energy for the STO- $(8+2) G$ basis is $E=-0.499996$ a.u. and $E^{-1}=-2.000015$ a.u. $^{-1}$ In the $R-R$ case, the convergence was too slow to be realistic, and in the $R-I$ case, the calculations were diverging.

\begin{tabular}{|c|c|c|c|c|c|c|c|}
\hline \multicolumn{6}{|c|}{$I-R$ case } & \multicolumn{2}{|c|}{ I-I case } \\
\hline Iteration & Energy & Iteration & Energy & Iteration & Energy & Iteration & Energy \\
\hline 1 & -0.449651 & 16 & -0.499751 & 31 & -0.499979 & 1 & -1.931726 \\
\hline 2 & -0.491420 & 17 & -0.499791 & 32 & -0.499982 & 2 & -1.943310 \\
\hline 3 & -0.497023 & 18 & -0.499824 & 33 & -0.499984 & 3 & -1.989179 \\
\hline 4 & -0.497824 & 19 & -0.499852 & 34 & -0.499986 & 4 & -1.996062 \\
\hline 5 & -0.498260 & 20 & -0.499875 & 35 & -0.499988 & 5 & -1.998332 \\
\hline 6 & -0.498542 & 21 & -0.499895 & 36 & -0.499989 & 6 & -1.999379 \\
\hline 7 & -0.498784 & 22 & -0.499912 & 37 & -0.499990 & 7 & -1.999758 \\
\hline 8 & -0.498981 & 23 & -0.499925 & 38 & -0.499991 & 8 & -1.999914 \\
\hline 9 & -0.499147 & 24 & -0.499937 & 39 & -0.499992 & 9 & -1.999975 \\
\hline 10 & -0.499285 & 25 & -0.499946 & 40,41 & -0.499993 & 10 & -1.999999 \\
\hline 11 & -0.499400 & 26 & -0.499955 & $42-44$ & -0.499994 & 11 & -2.000009 \\
\hline 12 & -0.499497 & 27 & -0.499961 & $45-49$ & -0.499995 & 12 & -2.000013 \\
\hline 13 & -0.499578 & 28 & -0.499967 & 50 & -0.499996 & 13 & -2.000014 \\
\hline 14 & -0.499646 & 29 & -0.499972 & & & 14 & -2.000015 \\
\hline 15 & -0.499703 & 30 & -0.499976 & & & & \\
\hline
\end{tabular}


fective and powerful method for formulating and calculating the exact wave function. This is the purpose of the present series of studies [2-6,38].

The Hamiltonian Krylov sequence is extended to include the inverse Hamiltonian, giving the inverse Krylov sequence. Since the Hamiltonian often involves Coulombic singularities, the element of the ordinary Krylov sequence, $H^{n} \psi_{0}$ with $n \geqslant 1$, strongly breaks the quantum-mechanical condition (i.e., integrable finiteness), while the inverse Krylov sequence does not have such a singularity problem. Combining the ordinary and inverse Krylov sequences, we obtain the complete Krylov sequence, which provides a natural basis for describing the exact wave function.

The SE and ISE concepts are combined to generalize the ICI method for calculating the exact wave function. Based on the variational principles in the regular and inverse forms, and on the $H$-square, inverse $H$-square, and cross- $H$-square equations, we formulated the four different cases of generalized ICI theory. They correspond to the two different formations of the variable operator $S$, depending on the use of $H$ or $H^{-1}$, and to the use of the two different variational principles, either regular or inverse. They have clear internal relations, and all four ICI methods give, in principle, the exact wave function. In these formulations, we used a positively shifted Hamiltonian for consistency. Further, we formulated the generalized ICI theory in a matrix representation, where a merit is that the definition of the inverse Hamiltonian is straightforward in comparison with the analytical case. The four different cases of ICI theory would have merits and demerits depending on the system, and the convergence rate would also be different, again depending on the system. In particular, when the Hamiltonian of the system has a singularity, the integrals involving higher-order products of the regular Hamiltonian may diverge and, therefore, the method may be problematic, but even in such a case, when we use the inverse Hamiltonian, we can calculate the exact wave function without having such a difficulty.

The generalized ICI theory has been applied to the hydrogen atom. Since the regular Hamiltonian has a Coulombic singularity at the origin, the ordinary ICI theory in the $R-R$ case is problematic: it involves the integrals that do not converge in the analytical formulation. Thus, even the hydrogen atom is a very good system to test the utility of the ISEbased concept. We have performed generalized ICI calculations in both analytical and matrix formulations and we could calculate the ground state of the hydrogen atom without the problem of singularity. The matrix formulation showed some interesting behaviors that are clearly attributable to the SE and SE concepts given in this paper.

In conclusion, the concept of the regular and inverse Hamiltonians and the various formulas originating from them seem to give an important insight for further studies aiming at the exact understanding of atomic and molecular systems.

\section{ACKNOWLEDGMENTS}

The author thanks Professor J. Cioslowski for some valuable conversations at the Girona Symposium and Dr. M. Hada, Dr. M. Ehara, and Dr. J. Hasegawa for some suggestions. This study has been supported financially by the special fund from the Ministry of Education, Science, Culture, and Sports.
[1] P. A. M. Dirac, Proc. R. Soc. London, Ser. A 123, 714 (1929).

[2] H. Nakatsuji, J. Chem. Phys. 113, 2949 (2000).

[3] H. Nakatsuji and E. R. Davidson, J. Chem. Phys. 115, 2000 (2001)

[4] H. Nakatsuji, J. Chem. Phys. 115, 2465 (2001).

[5] H. Nakatsuji, J. Chem. Phys. 116, 1811 (2002).

[6] H. Nakatsuji and M. Ehara, J. Chem. Phys. (to be published).

[7] T. Van Voorhis and M. Head-Gordon, J. Chem. Phys. 115, 5033 (2001).

[8] P. J. Knowles and N. C. Handy, Chem. Phys. Lett. 111, 315 (1984); G. L. Bendazzoli and S. Evangelisti, J. Chem. Phys. 98, 3141 (1993).

[9] Excellent review articles on the density-matrix approaches are found in Many-Electron Densities and Reduced Density Matrices, edited by J. Cioslowski (Kluwer Academic, New York, 2000).

[10] A. J. Coleman, Rev. Mod. Phys. 35, 668 (1963).

[11] A. J. Coleman and V. I. Yukalov, Reduced Density MatricesCoulson's Challenge, Lecture Notes in Chemistry Vol. 72 (Springer, Berlin, 2000).

[12] C. Garrod and J. Percus, J. Math. Phys. 5, 1756 (1964); L. J. Kijewski and J. K. Percus, Phys. Rev. A 2, 1659 (1970).

[13] L. J. Kijewski, Phys. Rev. A 6, 1659 (1972); 9, 2263 (1974); C. Garrod, M. Mihailovic, and M. Rosina, J. Math. Phys. 16, 868
(1975); M. V. Mihailovic and M. Rosina, Nucl. Phys. A 237, 229 (1975); C. Garrod and M. A. Fusco, Int. J. Quantum Chem. 10, 495 (1976); R. M. Erdahl, Rep. Math. Phys. 15, 147 (1979).

[14] M. Nakata, H. Nakatsuji, M. Ehara, M. Fukuda, K. Nakata, and K. Fujisawa, J. Chem. Phys. 114, 8282 (2001).

[15] M. Nakata, M. Ehara, and H. Nakatsuji, J. Chem. Phys. 116, 5432 (2002).

[16] H. Nakatsuji, Phys. Rev. A 14, 41 (1976); H. Nakatsuji, Theor. Chem. Acc. 102, 97 (1999).

[17] C. Valdemoro, in Density Matrices and Density Functionals, edited by R. Erdahl and V. Smith (Reidel, Dordrecht, 1987), p. 275.

[18] F. Colmenero, C. Perez del Valle, and C. Valdemoro, Phys. Rev. A 47, 971 (1993); F. Colmenero and C. Valdemoro, ibid. 47, 979 (1993); Int. J. Quantum Chem. 62, 369 (1994).

[19] H. Nakatsuji and K. Yasuda, Phys. Rev. Lett. 76, 1039 (1996); K. Yasuda and H. Nakatsuji, Phys. Rev. A 56, 2648 (1997); M. Ehara, M. Nakata, H. Kou, K. Yasuda, and H. Nakatsuji, Chem. Phys. Lett. 305, 483 (1999); M. Nakata, M. Ehara, K. Yasuda, and H. Nakatsuji, J. Chem. Phys. 112, 8772 (2000).

[20] D. A. Mazziotti, Phys. Rev. A 57, 4219 (1998); Chem. Phys. Lett. 289, 419 (1998); Int. J. Quantum Chem. 70, 557 (1998); Phys. Rev. A 60, 3618 (1999); Chem. Phys. Lett. 326, 212 
(2000); J. Chem. Phys. 115, 8305 (2001); 116, 1239 (2002).

[21] C. Valdemoro, L. M. Tel, and E. Pérez-Romore, Adv. Quantum Chem. 28, 33 (1997); Phys. Rev. A 61, 032507 (2000); K. Yasuda, Phys. Rev. A 59, 4133 (1999); D. A. Mazziotti, J. Chem. Phys. 115, 8305 (2001); D. A. Mazziotti and R. M. Erdahl, Phys. Rev. A 63, 042113 (2001).

[22] R. Kubo, J. Phys. Soc. Jpn. 17, 1100 (1962).

[23] W. Kutzelnigg and D. Mukherjee, J. Chem. Phys. 110, 2800 (1999); Chem. Phys. Lett. 317, 567 (2000); D. Mukherjee and W. Kutzelnigg, J. Chem. Phys. 114, 2047 (2001).

[24] M. Nooijen, Phys. Rev. Lett. 84, 2108 (2000).

[25] All equations of this group include the square of the Hamiltonian and/or the divided Hamiltonian. Previously, we also called this equation the H-nijou equation: "nijou" is Japanese meaning "square."

[26] O. Visser, P. J. C. Aerts, D. Hegarty, and W. C. Nieuwpoort, Chem. Phys. Lett. 134, 34 (1987); K. G. Dyall and K. Faegri, Jr., ibid. 201, 27 (1993).

[27] D. Andrae, Phys. Rep. 336, 413 (2000).

[28] D. Horn and M. Weinsten, Phys. Rev. D 30, 1256 (1984); see also R. Kosloff and H. Tal-Ezer, Chem. Phys. Lett. 127, 223
(1986); T. J. Park and J. C. Light, J. Chem. Phys. 85, 5870 (1986).

[29] J. Cioslowski, Phys. Rev. Lett. 58, 83 (1987).

[30] M. G. Marmorino, J. Chem. Phys. 114, 2513 (2001).

[31] H. Huang, Q. Xie, Z. Cao, Z. Li, Z. Yue, and L. Ming, J. Chem. Phys. 110, 3703 (1999); H. Huang, X. Zeng, and L. Ming, ibid. 112, 5257 (2000).

[32] For example, J. H. Wilkinson, The Algebraic Eigenvalue Problem (Oxford University Press, Oxford, 1965).

[33] W. Heisenberg, The Physical Principles of the Quantum Theory, translated by C. Eckart and F. C. Hoyt (Dover, New York, 1930).

[34] A. Messiah, Mécanique Quantique (Dunod, Paris, 1959).

[35] E. R. Davidson, Comput. Phys. Commun. 53, 1 (1989).

[36] W. E. Arnoldi, Q. Appl. Math. 9, 17 (1951).

[37] C. Lanczos, J. Res. Natl. Bur. Stand. 45, 255 (1950).

[38] H. Nakatsuji (unpublished).

[39] K. O-ohata, H. Taketa, and S. Huzinaga, J. Phys. Soc. Jpn. 21, 2306 (1966).

[40] P.-O. Löwdin, J. Chem. Phys. 18, 365 (1950). 